\title{
An Interactive Method of Characteristics Java Applet to Design and Analyze Supersonic Aircraft Nozzles
}

\author{
Thomas J. Benson ${ }^{1}$ \\ NASA Glenn Research Center, Cleveland, Ohio, 44135
}

\begin{abstract}
The Method of Characteristics (MOC) is a classic technique for designing supersonic nozzles. An interactive computer program using MOC has been developed to allow engineers to design and analyze supersonic nozzle flow fields. The program calculates the internal flow for many classic designs, such as a supersonic wind tunnel nozzle, an ideal 2D or axisymmetric nozzle, or a variety of plug nozzles. The program also calculates the plume flow produced by the nozzle and the external flow leading to the nozzle exit. The program can be used to assess the interactions between the internal, external and plume flows. By proper design and operation of the nozzle, it may be possible to lessen the strength of the sonic boom produced at the rear of supersonic aircraft. The program can also calculate non-ideal nozzles, such as simple cone flows, to determine flow divergence and nonuniformities at the exit, and its effect on the plume shape. The computer program is written in Java and is provided as free-ware from the NASA Glenn central software server.
\end{abstract}

\section{Nomenclature}

$\begin{array}{ll}a & =\text { speed of sound } \\ M & =\text { Mach number } \\ n & =\text { coordinate normal to streamline coordinate } \\ Q, R & =\text { Reimann invariants } \\ s & =\text { streamline coordinate } \\ u_{1,} u_{2} & =\text { velocities in } x_{1} \text { and } x_{2} \text { directions } \\ w & =\text { velocity magnitude in streamwise coordinates } \\ x_{1,} x_{2} & =\text { Cartesian coordinates } \\ \xi, \eta & =\text { characteristic coordinates } \\ \mu & =\text { Mach angle } \\ v & =\text { Prandtl-Meyer angle } \\ \theta & =\text { velocity direction in streamwise coordinates }\end{array}$

\section{Introduction}

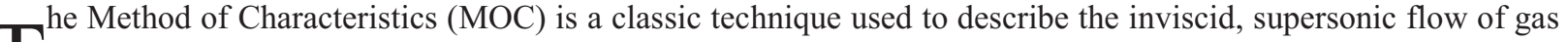
past objects. Details of the derivation and application of the technique are given in most undergraduate textbooks on compressible aerodynamics, such as Shapiro (Ref. 1) and Liepmann and Roshko (Ref. 2). A summary of the equations is given in the appendix of this paper. There are many references dating back to the 1950's and 60's, for example McCabe ( Ref. 3), that describe how the method can be used to design supersonic nozzles. There are two main practical nozzle design problems that use MOC: the design of a wind tunnel nozzle to provide shock free, uniform supersonic flow in the test section of the tunnel, and the design of a rocket nozzle which spends a majority of its flight time in the near-vacuum of space. The nozzle design is developed both numerically and graphically, since the method solves for both the values of flow variables, and the location at which the values occur relative to an initial flow field.

The current effort was prompted by NASA's Fundamental Aeronautics Program (FAP) and its High Speed (HS) project. One of the goals of the HS project is to reduce the sonic boom produced by a supersonic aircraft. A sonic boom has two main components: a shock off the nose and forebody of the aircraft, and a shock at the rear of the

\footnotetext{
${ }^{1}$ Senior Aerospace Engineer, Inlet and Nozzle Branch, 21000 Brookpark Road MS 5-11.
} 
aircraft. The strength of the rear shock is affected by the interaction of the supersonic exhaust from the engine nozzle and the supersonic free stream. The current effort attempts to model this interaction of the nozzle flow field and the supersonic flow at the rear of the aircraft and differs from previous studies in the requirement to model the flow internal to the nozzle, the flow in the plume issuing from the nozzle, the flow external to the nozzle, and the interactions between these three flows.

Special computer software, like Java and MATLAB, can solve the approiate differential equations that describe a specified flow problem and provide quick, first-order results. The input to and output from computer programs written in Java or MATLAB are principally graphical and visual, which make them perfect candidates for use with the MOC. Several professors and graduate students, (Devenport, Ref. 4), (Olsen, Ref. 5), (Khan, et.al., Ref. 6) have developed MOC solvers for university students. All of these MOC solvers use MATLAB as the computer language and all solve the classic rocket and wind tunnel nozzle problems. MATLAB is a commercial product from Mathworks, and is frequently provided to universities at a nominal license fee. Java is a product of SUN microsystems, now owned by Oracle, and is provided as free-ware that is bundled in the browser of the operating system for all personal computers. The author has been developing Java applets for aerospace students and practicing engineers for nearly 15 years, including FoilSim (Ref. 7), EngineSim, (Ref. 8), TunnelSys (Ref. 9) and several other applets to study compressible and hypersonic flows. All of the software programs and accompanying websites that describe the math and physics of the problems are available for free from NASA Glenn's central server as part of the Beginner's Guide to Aeronautics (BGA) (http://www.grc.nasa.gov/WWW/K-12/airplane/).

This paper describes the development and use of a new Java computer program that solves the MOC equations for a variety of supersonic nozzles. The program captures the classic designs of wind tunnel and rocket nozzles, but also solves for the plume and the interaction with the after-body flow for a supersonic aircraft. The program can be run in a design mode to develop nozzle contours, or in an analysis mode to evaluate off-design conditions. Simple geometries, like wedges and cones, can also be analyzed by the program. The program runs interactively within the Internet browser program of any personal desktop or laptop computer running Windows, MacOS, or Linux. Input to the program is accomplished using graphics widgets that will be described in the following section. Output is provided graphically in the form of a drawing of the nozzle and flow field and numerically in output boxes which are also described in the following section. The program is available for free from the NASA Glenn central server and can be downloaded and run off-line if the user prefers that mode of operation. With the download version, we provide a copy of the source code which the user can modify. Java applets restrict the reading and writing of files for security reasons. Consequently, the program is also available in a Java application version which runs native on the user's computer, requires some knowledge of Java applications, and the downloading of the Java Developer's Kit (JDK). The application can read and write files for the storage of data or for use with other applications and optimizers.

\section{MOCNozzle Computer Program}

The MOCNozzle computer program is being developed to provide practicing engineers and aerospace engineering students with a tool for the design and analysis of supersonic aircraft nozzles. Figure 1 is a screen grab of the current (June, 2014) version of the program and shows many of the features available to the user. The display is divided into three sections; top, bottom left, and bottom right. In the following figures, we shall show examples from several different test cases.

At the top of the figure is a schematic drawing of the nozzle and the solution flow field. The user can click on the drawing and move it within this drawing panel. The user can also vary the size of the display by using the "Zoom" widget at the far left or the "+" and "-_" buttons below the "Zoom" widget. If the user loses the image or wants to return to the initial size and orientation, one can click on the red "Find" button at the upper right and the image is returned to its initial position. At the upper left of the graphics panel are three buttons which control the appearance of the graphics. The default is to show the MOC "Mesh", as shown in Figure 1. In later figures, we will show examples of the "Plot" display. In Fig. 1 we have enabled the "Reflect" option which reflects the solution about the line of symmetry drawn on the figure. For most of the buttons used in the program, the current choice is shown as a yellow "lighted" button; the other possible choices are shown as blue buttons with white lettering. 


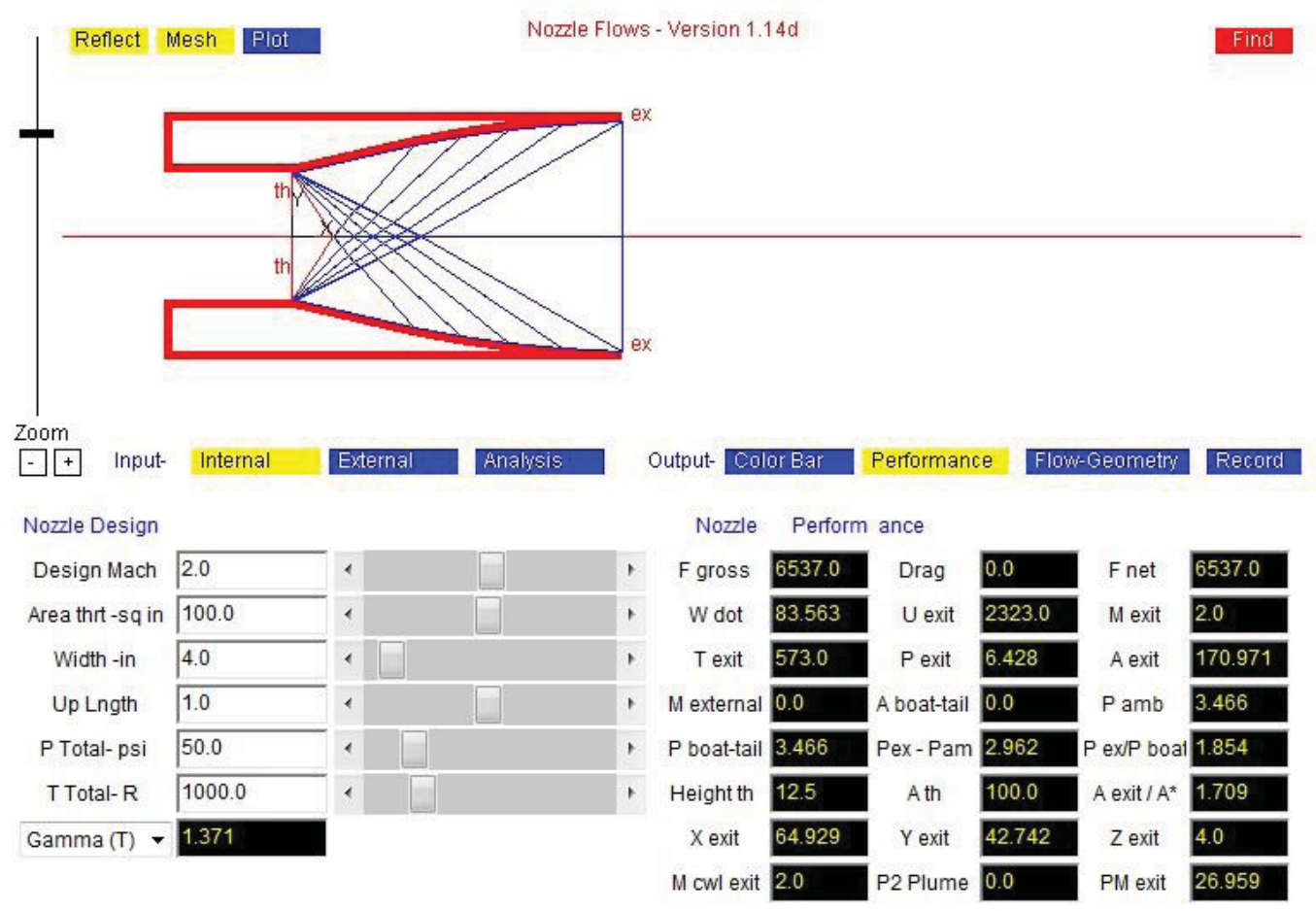

Figure 1. Ideal nozzle input and performance output panels.

At the lower left of the figure, we display the input panels for design and analysis of the supersonic nozzle. There are three possible input panels: one for the internal flow field, one for the external flow field, and one to control certain parameters used in the analysis. Figure 1 shows the "Internal" input panel which was selected using the buttons located above the panel. The user changes the value of an input variable by using the computer mouse to move the sliders, or the computer keyboard to type into the input boxes next to the sliders. Boxes with a white background and black letters signify variables which can be input or modified. On some input panels a drop-menu choice button is used to select an option. As the value of an input variable is changed, the program performs a new calculation and changes the nozzle schematic and the value of output variables on the output panel to the right.

At the lower right of the figure, we display the output panels. There are four possible output panels: one panel describes the performance of the nozzle as a whole, one panel provides details at selected locations in the flow field, one panel controls the color graphics used in the "Plot" display option, and one panel can be used to list the geometry of the nozzle internal contours so that a user may grab and record the results. Output is normally displayed in boxes with a black background and yellow, green, or cyan foreground. Figure 1 shows the "Performance" output panel for the particular case of an ideal nozzle with no external flow and no plume calculation. For this case, many of the external flow parameters have been set to zero and there is no external drag calculated. The flow is assumed to be choked at the throat, which establishes the mass flow rate (wdot) for the given input throat area, pressure and temperature. The input "Design Mach" number establishes the exit velocity, area, and exit static pressure, which determines the gross thrust.

The various input and output parameters shown in the figures of this paper have changed during code development and will be changed as newer versions of the computer program are developed and additional geometries are included. To obtain a current and accurate list of the flow variables used in the program, the reader should visit the following webpage on the NASA Glenn central software server: http://www.grc.nasa.gov/WWW/K12/airplane/mocnoz.html. The webpage contains the most current version of the program and includes detailed descriptions of all of the input and output variables used in the program. The webpage is intended to be a "User's Manual" for the program. Many of the input and output variables are hyperlinked to other webpages in the BGA for further explanation.

Figure 2 demonstrates some of the variations of input and output panels which a user can choose to display. The flow problem is the same as shown in Fig. 1, but in Fig. 2, we have not reflected the solution about the line of symmetry. The figure appears larger in the graphics window. In Fig. 2, we show the "Analysis" input panel with the "Problem" drop menu showing the current choices available for the user. The code can currently solve nine different 
nozzle geometry problems, with most problems having three possible flow solutions: internal flow only, internal flow plus plume flowing into a static gas, and internal flow plus plume flowing into a supersonic external flow. The ninth problem, an Axisymmetric Wedge Nozzle, is not shown on Fig. 2 because the standard drop menu widget only lists eight variables. On the analysis input panel of Fig. 2, we can select the number of rays used in the MOC analysis; increasing the number of rays increases the accuracy of the solution. The "Delx" parameter allows for a smoothing of the throat contour; a small value of "delx" produces a sharp corner at the throat, as shown in Fig. 2. The "\% Int Exp" parameter is used on plug nozzles to specify the amount of internal expansion.

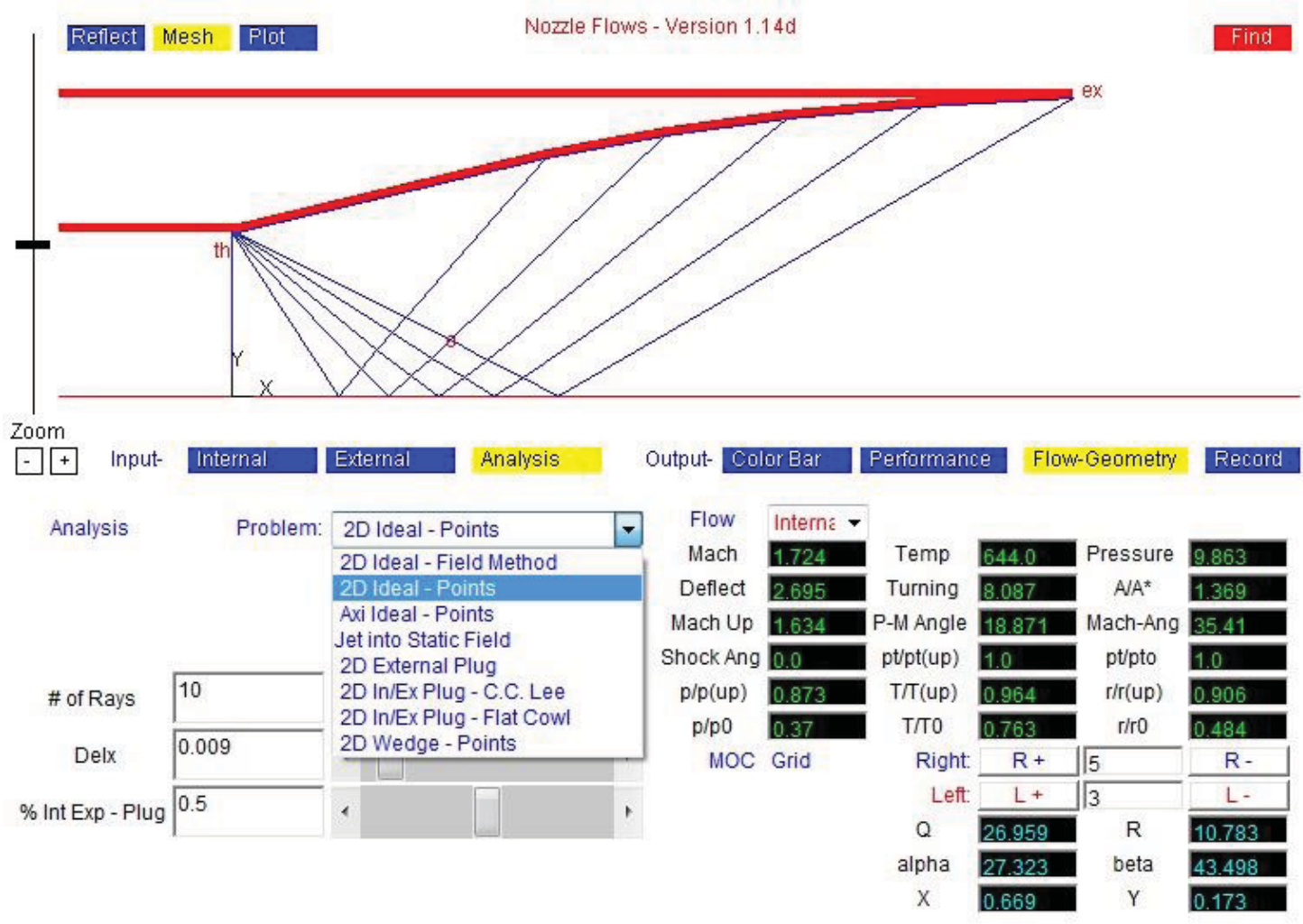

Figure 2. Analysis input and geometry output panels.

At the lower right of Fig. 2, we show the "Flow-Geometry" output panel. This panel is used in conjunction with the graphics panel to give a detailed look at the flow and geometry of the MOC grid. Right and Left running characteristic lines are selected using the $\mathrm{R}+/-$ and $\mathrm{L}+/$ - buttons at the lower right of the panel. The numbers of the left and right characteristics are shown in the boxes with the white background and the intersection of these characteristics is shown by a red circle on the graphic. At that point in the flow field ( $\mathrm{R}=5$ and $\mathrm{L}=3$ on Fig. 2), the values of pressure, temperature, flow turning, Mach number and other flow variables are displayed in the boxes on the panel. For reference purposes, $\mathrm{L}=1$ is the inner surface of the nozzle leaving the throat to the upper left and $\mathrm{R}=1$ is the line leaving the throat to the lower right. The " $\mathrm{X}$ " and "Y" location of the grid point relative to the throat and centerline are shown at the lower right along with the value of the Reimann invariants "Q" and " $\mathrm{R}$ " and two auxiliary angles "alpha" and "beta".

Figure 3 demonstrates some other variations of input and output panels which a user can choose to display. Figure 3 shows the flow through a plug nozzle with external supersonic flow and an exhaust plume. In Fig. 3 we have chosen to display color plots of the variables instead of the MOC mesh shown in the previous figures. For this figure, thirty right-running internal rays have been selected, so the flow field appears smoother than would occur with fewer rays. The variable chosen to be displayed in the plot and the range of variables is controlled by the "Color Bar" output panel at the right. The user can plot the pressure, temperature, Mach number, or flow turning angle by using the buttons in the center of the output panel. A color bar is displayed next to the choice buttons, with the maximum and minimum value displayed. The user can have the program survey the flow field to "Find" the maximum and minimum values by clicking on the button at the right of the panel. Or the user can input their own 
values of maximum and minimum and "Set" those values. To study the flow field more closely, the user could switch to the "Flow-Geometry" output panel shown in Fig. 2, while leaving the display in "Plot" mode. Also shown in Fig. 3 is the "External" input panel. For this particular case, we have a nozzle design that expands the flow from Mach = 1.0 in the throat to Mach =2.0 at the exit. The external flow is at Mach 2.3 free stream with a 6.55 degree boat-tail that expands the external flow to near Mach 2.7 and the pressures from the internal flow and the external flow do not match at the exit. To produce matched pressures at the plume boundary, a shock wave is thrown into the external flow and an expansion fan is generated into the internal flow. A slip line is formed between the internal plume and the external flow as shown by the black line on the graphic. The shock wave generated at the nozzle exit contributes to the sonic boom signature of this particular nozzle design. With the software, we can readily examine methods to reduce the strength of this shock wave.

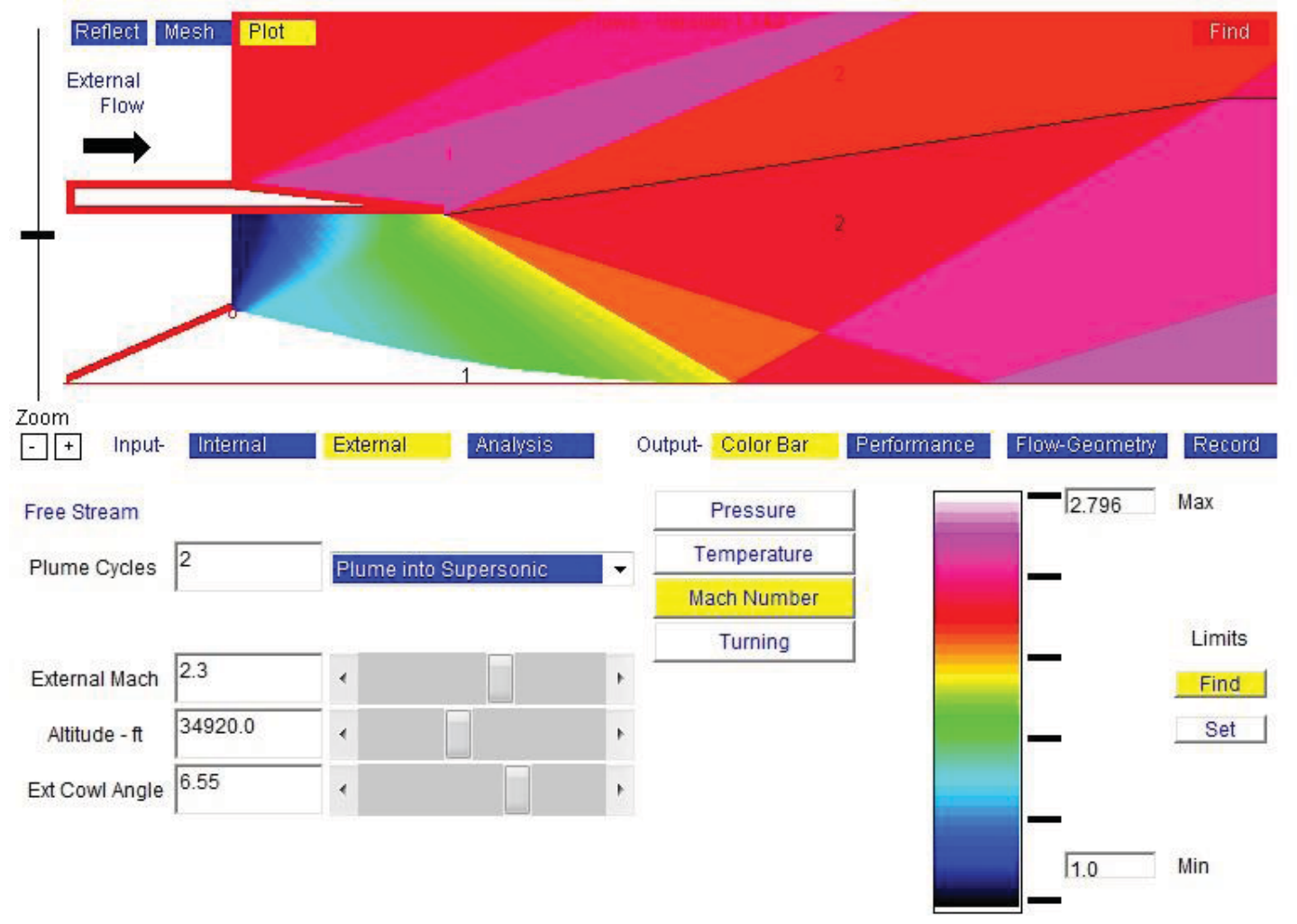

Figure 3. External input and color bar output panels.

The previous three figures have illustrated some of the many options available in the MOCNozzle Java applet. Each of these cases were run in design mode, in which the user specified some design constraints (exit Mach number, area at the throat, total pressure in the throat, etc.) and the MOC was used to produce a contour of the nozzle that would produce uniform flow at the exit of the nozzle to meet those constraints. Conditions of the uniform flow may not match the flow conditions in the external flow, which leads to shocks and expansions being generated in the plume and external flows. These flows are also computed by the method of characteristics for the expansion fan, and application of the shock relations from NACA 1135 (Ref. 10) when a shock is present.

In Fig. 4, we show a different type of problem. The geometry of the nozzle is now specified as a simple, straight-sided 2D wedge with a variable wedge angle (for the case shown, wedge angle $=5.88$ degrees). The flow problem involves calculating the flow field to produce a non-uniform exit flow that averages Mach $=2.0$. An expansion fan is generated at the throat of the nozzle and the fan is reflected from the centerline of the nozzle back onto the surface and in turn back onto the centerline. Flow throughout is determined from an MOC analysis. Comparing this result with Fig. 2, we note that the exit flow is uniform and aligned with the free stream for the ideal nozzle of Fig. 2, but the exit flow is not uniform for the simple 2D wedge nozzle of Fig. 4.

The 2D wedge nozzle exit flow field has varying degrees of divergence at the exit as well. The determination of the gross thrust of this nozzle includes the effects of flow divergence. While this type of nozzle is easier to manufacture than an ideal nozzle, there are performance penalties which can be evaluated by using this software. 


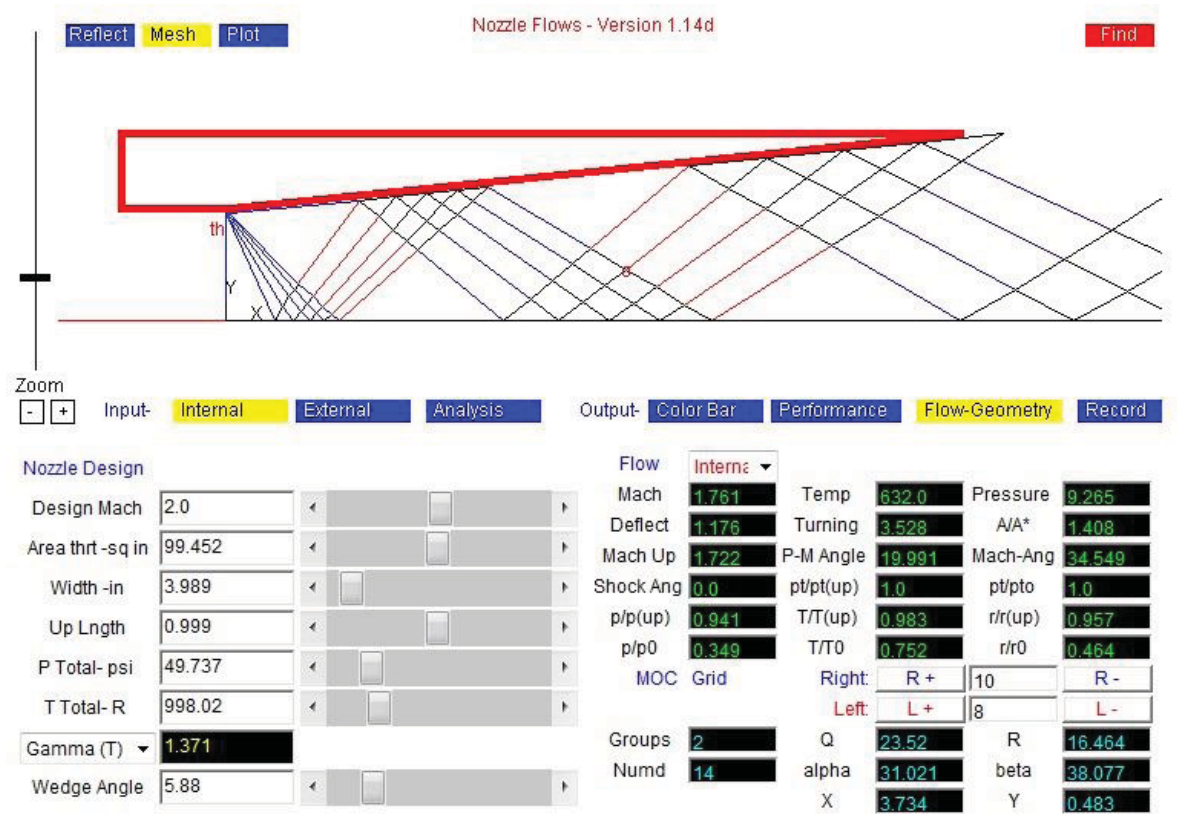

Figure 4. Analysis of simple 2D wedge nozzle.

Figure 5 shows the computed flow field for an axisymmetric nozzle exhausting into stagnant air. This flow field is similar to the flow in Fig. 4 except the geometry is axisymmetric and the exhaust plume is included. The display in Fig. 5 shows color contour plots of the Mach number as opposed to the MOC grid of Fig. 4. It is apparent that the exit flow field is non-uniform because of the reflection of expansion fans from the straight walls of the axisymmetric nozzle. The walls are not contoured to cancel out impinging expansion waves. The non-uniformity persists throughout the exhaust plume. When coupled with an external flow field, we will be able to determine the effects of non-uniformity on the formation of shock waves in the external flow field.

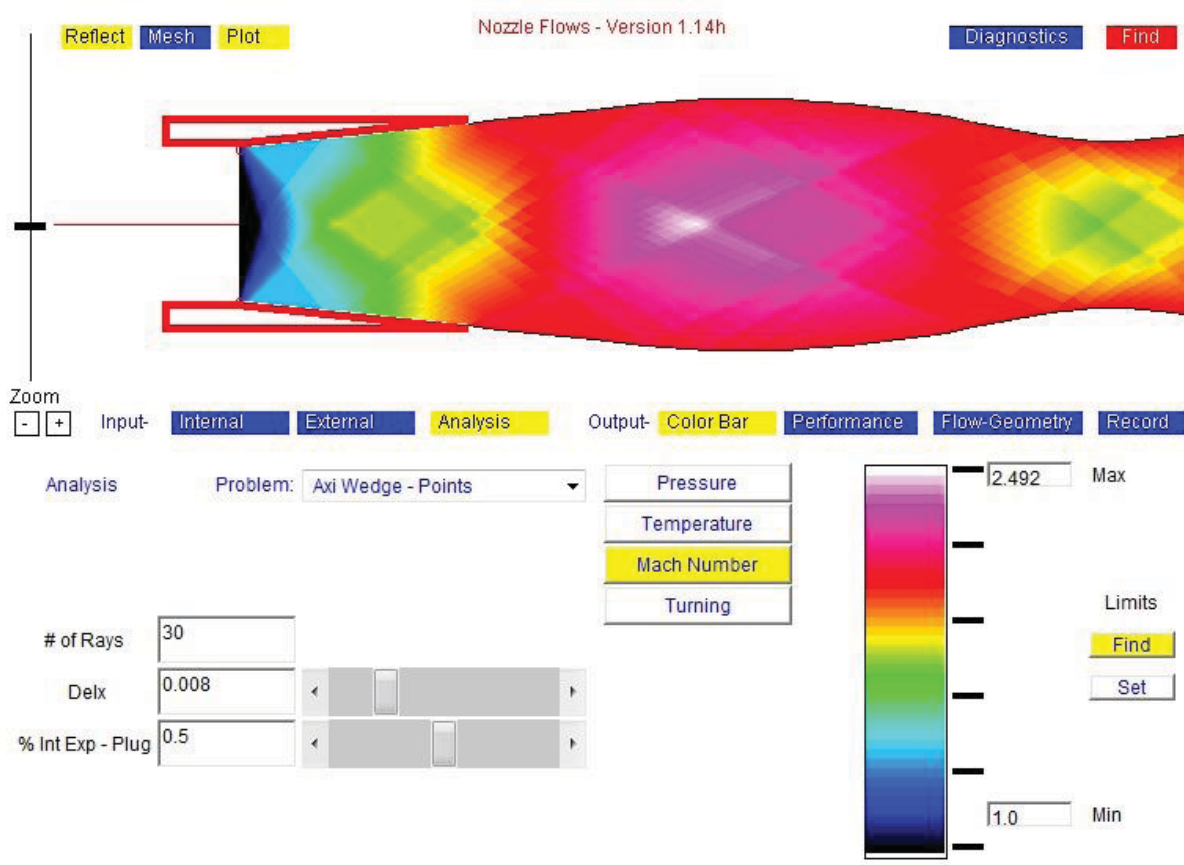

Figure 5. Analysis of axisymmetric wedge nozzle. 


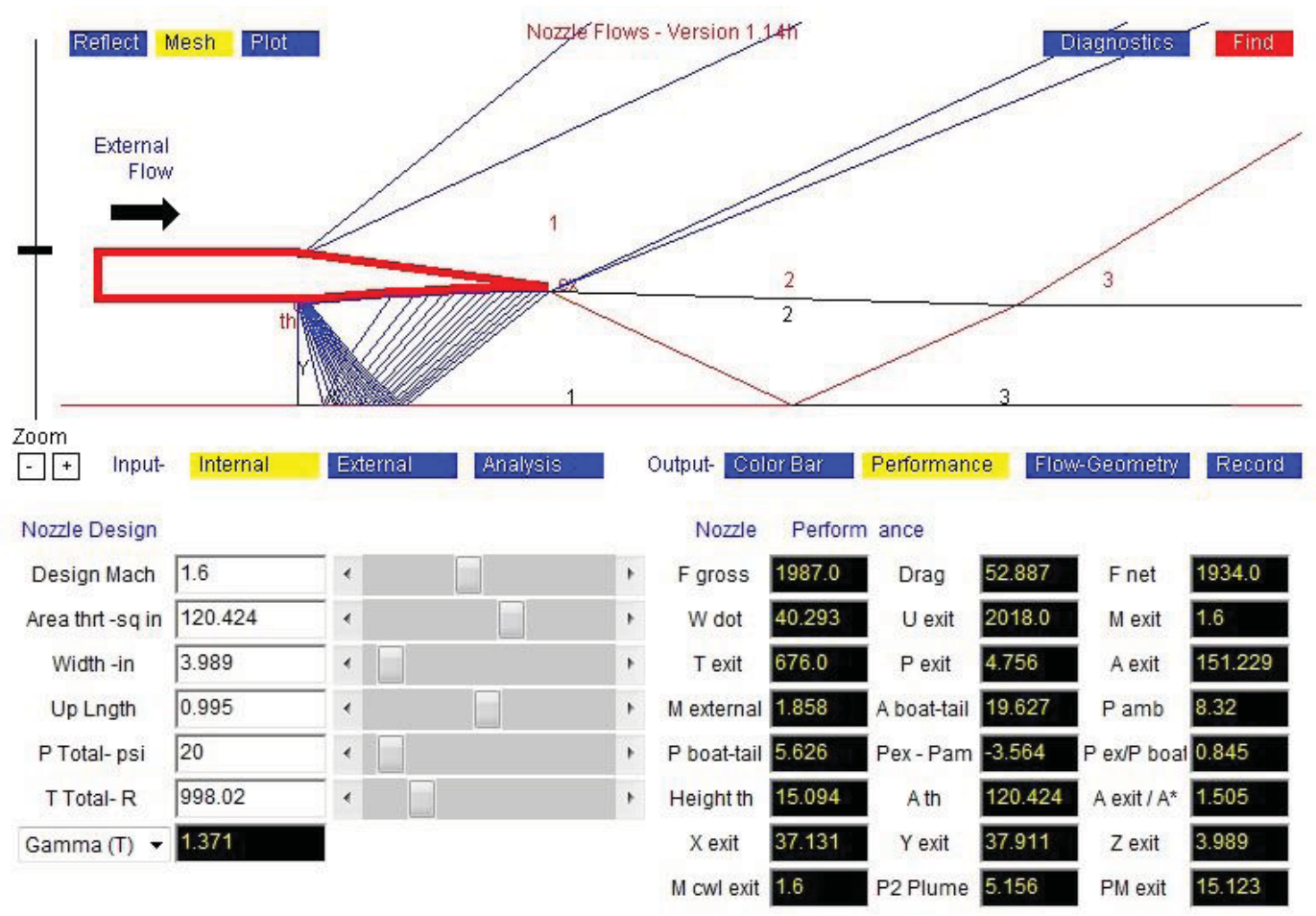

Figure 6. Interaction of internal, external and plume flows.

Figure 6 demonstrates the use of the computer program to model the internal, external and plume flows of a supersonic aircraft nozzle. For this test case, a Mach 1.6 nozzle exit speed was specified and the external flow was also specified at Mach 1.6 at 15,000 feet. A 7.5 degree external flow angle was specified, which accelerated the external flow to Mach 1.858 at the nozzle exit. The nozzle total pressure was then adjusted to produce a slightly over-expanded nozzle; the exit static pressure at end of the internal nozzle is slightly less than the static pressure produced by the external surface of the nozzle. As a result, a shock wave was generated in the plume and a small expansion fan was generated into the external flow. The shock wave crosses the plume and reflects from the centerline of the nozzle as shown by the red lines between regions 1-2 and 2-3 at the aft of the nozzle. A slip plane is generated between the plume flow and the external flow as shown as a black line from the exit of the nozzle. The static pressure is matched across the slip plane but the Mach number and velocity are different. As the shock wave in the plume crosses the slip plane it is deflected slightly to the left towards the nozzle. The shock wave will eventually interact with the two expansion fans in the external flow at a large distance from the nozzle. The interactions of the expansion fans with the shock will, in theory, decrease the strength of the shock and reduce the sonic boom strength. In future versions of the computer program, we will calculate and display the interaction far from the nozzle centerline. There is a performance penalty (lower gross thrust) that results from this nozzle design because of the over-expanded operation. With the software, we can quickly assess the amount of this penalty, to first order accuracy.

\section{Future Work}

This paper has presented a new computer program for interactively designing and analyzing the performance of a supersonic nozzles. The program enables a computation of the internal flow through the nozzle, the external flow at the afterbody of the aircraft, and the plume produced by the nozzle and the interaction between the three flow fields. This is the first release of the program and while it can model a variety of aircraft nozzle designs, there are several other designs that we intend to include in future releases of the program. The first additions will be a 2Dwedge plug nozzle and an axisymmetric cone plug nozzle. There has also been a request from the HS project to include a three stream plug nozzle. During code development, we have been comparing the results of test calculations to other MOC computer programs to detect and eliminate coding errors. We have also compared with 
analytic calculations where appropriate. In the next phase of code validation we will identify and compare the results of calculations to experimental results. Future papers will document these validation efforts.

\section{Conclusion}

High speed personal computers coupled with interactive operating systems and computer graphics provide many new opportunities for solving fluid mechanics problems. This paper describes a new interactive computer program developed at the NASA Glenn Research Center for NASA's Fundamental Aero Program. The software uses the Method of Characteristics to interactively design and analyze supersonic flow through a jet aircraft nozzle. The program is written in Java and is provided as free-ware from the NASA Glenn Educational Program's Beginner's Guide to Compressible Flow website. The code differs from other MOC solvers in the variety of problems that it can solve, and in its ability to simultaneously model the internal flow, external flow, jet plume, and interactions between the three flow fields. The goal is to gain a better understanding of the generation of shock waves by the nozzle/afterbody of a supersonic jet aircraft in order to lower the sonic boom signature. The program is available in three forms: as an on-line Java applet from the NASA Glenn central server, as a downloaded off-line applet that runs in any browser, and as an off-line Java application. When using the application version, the user must download and install the Java Developer's Kit (JDK) from Oracle which is provided as free-ware. The application version reads an optional input file and prints an output file and may be bundled with an optimizer to analyze a series of nozzle designs. The program is currently being validated by comparison with experimental data.

\section{Appendix}

Following the derivation in Leipmann and Roshko (Ref. 2), the full nonlinear equations of motion for twodimensional, nonviscous, irrotational flow are

$$
\begin{gathered}
\left(u_{1}^{2}-a^{2}\right) \frac{\partial u_{1}}{\partial x_{1}}+u_{1} u_{2}\left(\frac{\partial u_{1}}{\partial x_{2}}+\frac{\partial u_{2}}{\partial x_{1}}\right)+\left(u_{2}^{2}-a^{2}\right) \frac{\partial u_{2}}{\partial x_{2}}=0 \\
\frac{\partial u_{2}}{\partial x_{1}}-\frac{\partial u_{1}}{\partial x_{2}}=0
\end{gathered}
$$

For $\left(u_{1}^{2}+u_{2}^{2}\right) / a^{2}>1$, the equations are hyperbolic in nature and may be solved by the method of characteristics. The method re-casts the equations in natural coordinates. The velocities are expressed in terms of magnitude and direction $(w, \theta)$ and the independent variables are the streamline coordinates $(s, n)$. The recast equations are:

$$
\begin{aligned}
& \frac{\cot ^{2} \mu}{w} \frac{\partial w}{\partial s}-\frac{\partial \theta}{\partial n}=0 \\
& \frac{1}{w} \frac{\partial w}{\partial n}-\frac{\partial \theta}{\partial s}=0 \\
& \cot ^{2} \mu=M^{2}-1
\end{aligned}
$$

If we introduce the Prandtl-Meyer function, $v$, as defined by:

$$
d v=\cot \mu \frac{d w}{w}
$$

Then Eq. 2 reduces to: 


$$
\begin{aligned}
& \frac{\partial v}{\partial s}-\tan \mu \frac{\partial \theta}{\partial n}=0 \\
& \tan \mu \frac{\partial v}{\partial n}-\frac{\partial \theta}{\partial s}=0
\end{aligned}
$$

There is a compatibility relation that exists between $v$ and $\theta$ along characteristic Mach lines. The Mach lines are inclined to the streamlines at the angles $+/-\mu$. If we define coordinate lines $\xi$ and $\eta$ along the Mach lines, the compatibility relation reduces to:

$$
\begin{aligned}
& \frac{\partial}{\partial \eta}(v-\theta)=0 \\
& \frac{\partial}{\partial \xi}(v+\theta)=0
\end{aligned}
$$

Integration of Eq. 6 leads to :

$$
\begin{aligned}
& v-\theta=R \quad \text { a constant along an } \eta \text { characteristic } \\
& v+\theta=Q \quad \text { a constant along a } \xi \text { characteristic }
\end{aligned}
$$

The solution of these equations are determined by specifying boundary conditions at a starting plane and along the edges of the flow field. The shape and location of the characteristic lines are part of the solution. When axisymmetric coordinates are used, there are some additional terms in the equations of motion that depend on the geometric location of the characteristics relative to the axis. The equations cannot be easily integrated as shown above and the solution is obtained numerically.

\section{Acknowledgments}

Development of the MOCNozzle applet has been supported by the High Speed project of the Fundamental Aeronautics Program. The software is made available through the Beginner's Guide to Compressible Flow which is a product of the NASA Glenn Educations Programs Office and is supported by NASA HQ. The most current version of the program is available at:

\section{http://www.grc.nasa.gov/WWW/K-12/airplane/mocnoz.html}

\section{References}

${ }^{1}$ Shapiro, A.H., The Dynamics and Thermodynamics of Compressible Fluid Flow, Ronald Press Co., New York, 1953.

${ }^{2}$ Liepmann, H.W. and Roshko, A., Elements of Gasdynamics, John Wiley \& Sons, Inc., London, 1957.

${ }^{3}$ McCabe, A., "Design of Supersonic Nozzle", Aeronautical Research Council, R\&M 3440, 1967.

${ }^{4}$ Devenport, W.J., "Method of Characteristics for Two-Dimensional Isentropic Supersonic Flows", Matlab Functions and Applications Scripts for Educational Use. Department of Aerospace and Ocean Engineering, Virginia Tech, 2009.

${ }^{5}$ Olsen, B.J., “2-D Nozzle Design”, MATLAB Central, Mathworks 2007.

${ }^{6}$ Khan, M.A, Sardiwal, S.K., Sharath, M.V., and Chowdary, D.H., "Design of a Supersonic Nozzle using Method of Characteristics", IJERT, Nov, 2013.

${ }^{7}$ Benson, T.J., "Using Computers in Fluids Engineering Education,", ASME 1998 Fluids Engineering Summer Meeting, ASME Paper FEDSM98-5135, 1998. 
${ }^{8}$ Benson, T.J., "EngineSim and the Beginner's Guide to Propulsion", ASME 2001 Fluids Summer Meeting, Paper FEDSM2001-18228, 2001.

${ }^{9}$ Benson, T.J., Galica, C.A., and Vila, A.J., “Beginner's Guide to Wind Tunnels with TunnelSim and TunnelSys", AIAA Paper 20100080, 2010.

${ }^{10}$ Ames Research Staff, Equations, Tables, and Charts for Compressible Flow, NACA Report 1135, 1953. 